\title{
Male genital variation in a moth Pammene luedersiana (Lepidoptera: Tortricidae)
}

\author{
MARKo MUTANEN ${ }^{1}$, SEPpo RYTKÖNEN ${ }^{1}$, JARI LINDÉN² and JANNE SINKKONEN ${ }^{3}$ \\ ${ }^{1}$ Department of Biology, P.O. Box 3000, FIN-90014 University of Oulu, Finland; e-mail: marko.mutanen@oulu.fi \\ ${ }^{2}$ Postikatu 10 C 6, 08100 Lohja, Finland \\ ${ }^{3}$ Vilniementie 4 C 11, 02940 Espoo, Finland
}

Key words. Lepidoptera, Tortricidae, geographic variation, geometric morphometrics, genital evolution, genitalia, genital variation, Pammene luedersiana

\begin{abstract}
Insect genital characters are extensively used in species level taxonomy, and their value in species delimitation is great. Based on the lock-and-key hypothesis and that genital differences function as a mechanical isolation system between species, the value of genital characters has been thought to be superior to non-genital characters. Although geographical and other kind of intraspecific variation of genitalia is often assumed very moderate, its real extent is insufficiently investigated. We examined patterns of morphological variation in the male genitalia of the tortricid moth Pammene luedersiana, using geometric morphometric tools including thin-plate spline deformation grids, and found significant variation. This variation is continuous both within and between populations. No systematic shape variation was observed between populations, but genital size showed some geographic variability. The results suggest that genital morphology is not constant and should therefore be used with caution in lepidopteran taxonomy.
\end{abstract}

\section{INTRODUCTION}

Insect genital characteristics have routinely been used to delimit species as well as in other fields of systematics for decades. The extensive use of genital characteristics is simply due to their usefulness: genitalia very often provide the only way to distinguish species. Consequently, identification of externally similar species typically demands dissection of their genitalia. It could also be argued that in many insect taxa genitalia have a superior value in taxonomy. They have been assumed to vary less than non-genital characters on a local, but presumably also on a broader geographic scale. Taxa showing consistent differences in genital morphologies are regularly considered distinct species regardless of the extent of other differences or biogeographical patterns between the taxa. The idea that a certain structure could provide a universal way in species delimitation sounds appealing to morphologists, but in the light of current knowledge about the evolution of genital morphology, it remains unsatisfactorily supported. Overall, genital variability across the diversity of insect taxa has been insufficiently investigated.

Underlying philosophical rationales for the applicability of genital characters have seldom been discussed in a taxonomic context, but the evolutionary causes of rapid and divergent evolution of genital morphology have been of great interest among evolutionary biologists (for reviews, see Eberhard, 1985; Shapiro \& Porter, 1989; Arnqvist, 1997; Hosken \& Stockley, 2004). The first explanation for the phenomenon was presented by Dufour (1844). He observed that certain fly species regularly have species-specific genital morphology, and explained this as a function of mechanical isolation between species. This idea, later termed the lock-and-key hypothesis, was adopted by many taxonomists (Eberhard, 1985), and the post-Darwinian version of it was reviewed by Shapiro \& Porter (1989). The Darwinian lock-and-key hypothesis explains genital divergence as a form of character displacement. There is, however, little empirical or experimental evidence for the hypothesis, and current understanding is that genital evolution is driven mostly by sexual selection (Arnqvist, 1998; Hosken \& Stockley, 2004).

Sexually selected traits usually vary and there are studies showing that genital characters may indeed vary considerably (e.g. Arnqvist \& Thornhill, 1998; House \& Simmons, 2003; Mutanen, 2005; Mutanen et al., 2006). There are no evolutionary reasons to suppose that this variation could not show geographical effects. Instead, one can assume that genitalia may show both gradual and discrete variation in space. Observations of discrete variation in genitalia between geographically isolated populations are scanty, but this may also partly be due to circular reasoning (see Shapiro \& Porter, 1989): genitally polymorphic species are not likely to be found when species are defined by their genitalia (Mutanen \& Kaitala, 2006). Similarly, providing that sexual selection is responsible for genital evolution, there are no grounds to assume that genital shape would be independent of juvenile conditions and hence of body (or overall genital) size (Arnqvist, 1997).

The extent of variation in genital morphology is often discussed in taxonomic revisions, usually based on a subjective non-quantitative visual evaluation. If clear-cut qualitative differences are lacking or there is much intraspecific variation, the delimitation may traditionally have 
been further supported with distance measurement statistics of quantitative traits. Unfortunately, this method collects mostly information on size, but limitedly on shape (Zelditch et al., 2004). The value of size should not be undervalued, particularly as genital size has been shown to generally vary less than body size (e.g. Eberhard et al., 1998). In practice taxonomists usually favor characters that are invariant to size, that is, differences between shapes of structures. However, using conventional distance measurements and appropriate statistics the components of size and shape cannot be separated completely (Zelditch et al., 2004). For example, although the first component of the Principal Component Analysis (PCA) is usually interpreted as the size factor and the second as the shape one, there is at least some shape present in the former and size in the latter (Zelditch et al., 2004). In addition, the shape differences are usually difficult to interpret in conventional PCA.

Recent revolutionary advance in shape quantification (Rohlf \& Marcus, 1993; Adams et al., 2004), usually referred to as geometric morphometrics, uses geometric coordinates of landmarks of structures. The method is based on Kendall's definition of geometric shape and his theory of shape spaces (Kendall, 1977, 1984). According to Kendall (1977), shape is all the geometric information that remains when the effects of location, scale and rotation are filtered from the object. The fundamental advantage is that the methods based on Kendall's shape spaces enable visualizing the shape changes between the objects with so-called thin-plate spline deformations (Bookstein, 1991). For taxonomists, the opportunity to calculate and visualize average shapes for species and the limits of localized intra- and interspecific variation is of particular importance.

Pammene luedersiana (Sorhagen, 1885) (Lepidoptera, Tortricidae) is a small tortricid moth. We investigated variation in the male external genital morphology of $P$. luedersiana using geometric morphometric tools. More specifically, we studied whether the size and shape variation in genitalia of $P$. luedersiana shows discontinuities in local and geographic scales. Second, we explored whether there is correlation between genital size and shape. We discuss our findings in both evolutionary and systematic contexts.

\section{MATERIAL AND METHODS}

\section{Samples}

The material of 131 male specimens of $P$. luedersiana was mainly collected with pheromone traps, but several specimens were also gathered from public and private collections. A great majority of specimens were fresh (collected 1995-2005), some were slightly older but only a couple museum specimens were more than fifty years old. All specimens were collected from Southern and Central Finland, North Europe (Fig. 1). In some localities, only one or a couple of specimens were obtained, but in four geographically distinct areas, larger series proper for statistical testing (see below) were included. In pheromone traps, we used a commercial Cydia funebrana pheromone, which attracts not only that pest species, but also several related species, including our target $P$. luedersiana.

\section{Genital dissection and landmark digitation}

The abdomens of pinned specimens were cut off and boiled in $10 \%$ caustic potash. The rigid genitalia were then removed from the surrounding tissues, cleaned, stained with Eosin Red and dehydrated with ethanol. The genitalia were mounted on microscope slides using standardized amount (one drop) of Euparax fixative. The male clasping genital structures, valvae, were spread in a standard position across all specimens. Since valvae are rigid and flat, little preparation distortion is assumed by this operation. The male genitalia were photographed using Olympus C-7070 Wide Zoom digital camera and processed with the TPSUTIL 1.26 program (Rohlf, 2004a). The landmark digitations were conducted with the TPSDIG 1.40 program (Rohlf, 2004b). Given the fact that male genital shape in moths is subject to large interspecific variation, a relatively low number of easily definable landmarks is usually found. Frequently, there are diagnostic features in shape of male valva outline. For this reason, we used a combination of landmarks and so-called sliding semilandmarks in the analyses. Sliding semilandmarks are appropriate for outline shape quantification and they can be combined with true landmarks in a single analysis (Bookstein, 1997). Sliding semilandmarks are allowed to slide along an outline curve to optimally fit with the corresponding landmarks of the reference (consensus) shape. After sliding, they are analyzed together with true landmarks. We digitized a total of 19 landmarks of the right valva. Four of them (landmarks 1, 8, 14 and 19) were assigned as landmarks and the rest as sliding semilandmarks. Since we investigated outline shapes, all landmarks and semilandmarks were placed on the outline and in a density that sufficiently covered the outline shape (Fig. 2). For convenience, both landmarks and semilandmarks are below referred to as landmarks.

\section{Digitizing error}

All morphometric data is affected at least by some inaccuracy in measurements. Measurement error makes real patterns more obscure and consequently more difficult to detect. Measurement error cannot be completely eliminated, but its effect can be reduced and evaluated. Evaluating measurement error is important for two reasons. First, it enables evaluation of the relative magnitude of measurement inaccuracy. Second, it increases the statistical power of the analyses (Arnqvist \& Mårtensson, 1998). The measurement error of geometric morphometric data consists of methodological inaccuracy (distortion in specimen preparation), instrumental error (optical distortions) and inaccuracy in landmark digitations (Arnqvist \& Mårtensson, 1998).

No optical system is free of distortions, but they can be minimized using complex lens systems, and therefore the effects of instrumental error are best reduced by using optically good equipment. In small objects such as insect genitalia, the morphometric examination usually requires the objects to be permanently mounted in a fixative between microscope slides, making the evaluation of preparation error practically impossible. In this study, we repeated all digitations twice and evaluated digitation accuracy by calculating percentual digitation error as follows (Yezerinac et al., 1992):

$\% \mathrm{DE}=\frac{\mathrm{S}_{\text {within }}^{2}}{\mathrm{~S}_{\text {within }}^{2}+\mathrm{S}_{\text {among }}^{2}} \times 100$

where $\mathrm{s}^{2}$ within is within-group mean square ( $\left.\mathrm{MS}_{\text {within }}\right)$ and $\mathrm{S}^{2}$ among is calculated from equation $\left(\mathrm{MS}_{\text {among }}-\mathrm{MS}_{\text {within }}\right) / \mathrm{m}$, in which $\mathrm{MS}_{\text {among }}$ is between-group mean square and $\mathrm{m}$ is the number of measurements.

We summed $\mathrm{S}_{\text {within }}^{2}$ and $\mathrm{S}^{2}$ among over all landmarks. This is justified, because geometric shape is understood as a configuration of landmarks, but also necessary, because variation of a par- 


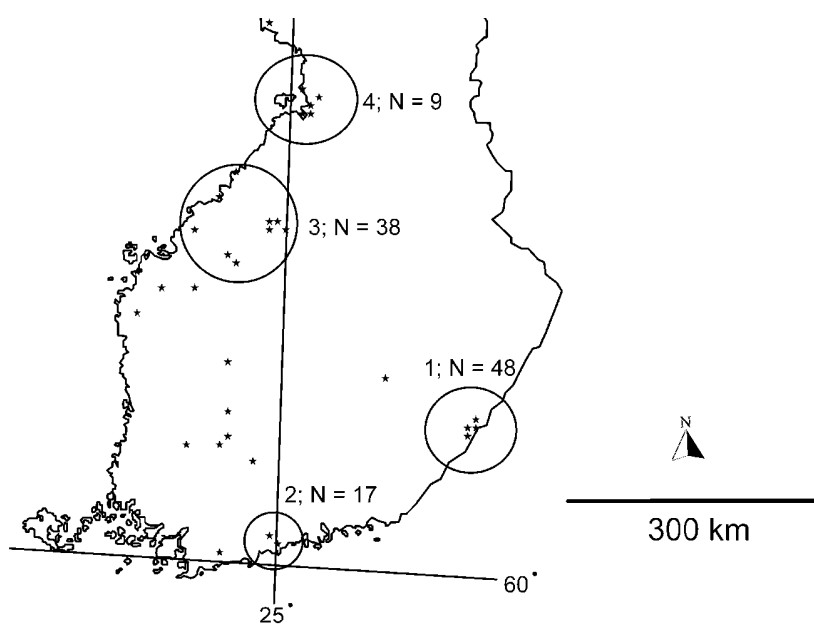

Fig. 1. Sample localities and sample areas (1-4) used in tests of geographical effects in genital size and shape variation (circled). Number of individuals from each area are indicated in the map.

ticular superimposed landmark is not independent from variation of other landmarks.

\section{Genital size and shape variation}

Genitalia sizes were quantified by using the centroid size, the square root of the sums of squared distances of a set of landmarks from their centroid, and it is a good estimate of overall size (Zelditch et al., 2004). In geometric morphometrics, the centroid size is used to scale configurations of landmarks to unit centroid size. We tested whether there are geographic patterns in genital size using ANOVA, with individual centroid size as a dependent variable, sample area and side as fixed factors and measurement as a random factor. Only larger series of individuals from four adequately distinct geographical areas were included in this test (Fig. 1). Centroid sizes were calculated using the TPSRELW 1.35 program (Rohlf, 2003).

In shape investigation, the landmark coordinate data was subjected to generalized least-squares Procrustes superimposition (Rohlf \& Slice, 1990). Again, the superimposition was carried out using TPSRELW 1.35 program (Rohlf, 2003). This action filters all the non-shape variation (variation in size, position and orientation) from the data. Since the digitizing error was small (see results), we calculated the average coordinates for aligned landmarks and used those in subsequent analyses. This reduces the number of analyses, but more importantly, also increases the statistical power by reducing the impact of digitizing error (Arnqvist, 1998). Given that the errors are rather homogeneous over measurement points, this is a convenient way to simplify the analyses and still maintain their statistical power. For statistical analyses, we calculated partial warp scores for the aligned coordinates of specimens. Partial warps describe the net local information in a deformation along each coordinate axis, and define the location (coordinates) of each specimen in the shape space. Partial warp scores can therefore be interpreted as shape variables and they are used to construct the thin-plate spline visualization of shape changes. Furthermore, a complete set of partial warp scores (uniform component included) is appropriate for various multivariate statistical analyses. Since the number of partial warp scores is usually high and the shape change therefore difficult to interpret, we ran a PCA of partial warp scores for shape investigation. The distribution of shape variation was investigated using PCA plots and the most significant shape changes visualized with thin-plate spline deformation grids. The

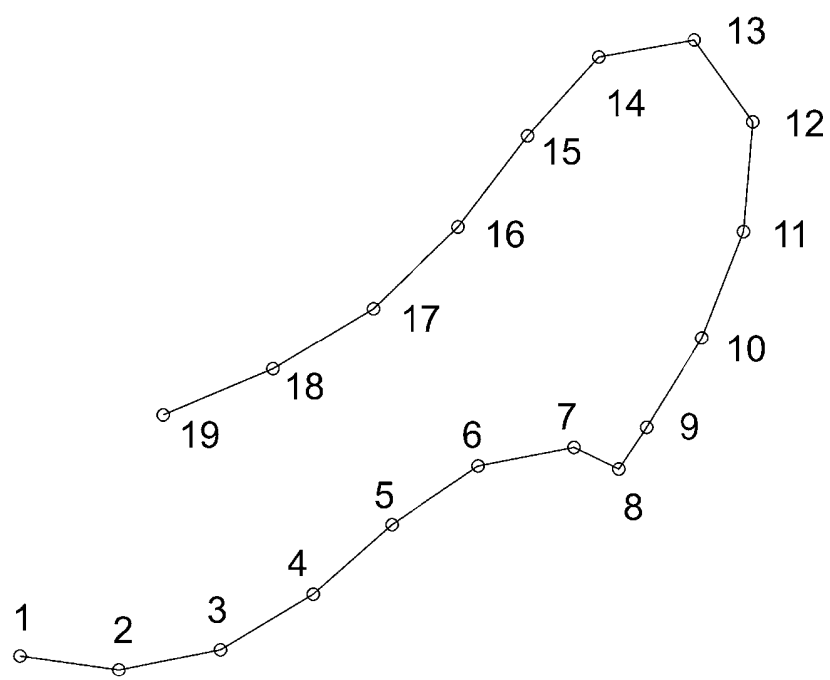

Fig. 2. Landmarks $(1,8,13,19)$ and sliding semilandmarks $(2-7,9-12,14-18)$ used to analyze size and the outline shape of male right valvae of $P$. luedersiana.

geographic variability was tested using MANOVA with the complete set of partial warps scores as dependent variables and sample area as a fixed factor. This analysis was done using the average landmarks (repeated measurements combined) of the right side of the valvae.

\section{Correlation between genital size and shape}

Since we detected clear variation in genital size, we tested whether this variation is correlated with variation in shape. This was done with regression analysis with averaged centroid size of the right side as an independent factor and the first three principal components of the right side, altogether explaining clear majority of all observed shape variation (see results), as dependent factors.

\section{RESULTS}

\section{Digitation error}

Only $0.20 \%$ of all observed size variation was due to digitation inaccuracy. Since centroid size is calculated based on sums of squared distances of all landmarks from their centroid, it is not very sensitive to (random) inaccuracy in individual digitations. Digitation error in genital shape quantification was $3.13 \%$ of all shape variation. Both size and shape digitation errors were considered low, and for this reason we used averaged values in further analyses.

\section{Genital size and shape variation}

The ANOVA revealed that the centroid size of genitalia differs between localities $\left(\mathrm{F}_{3,3}=2748.44, \mathrm{P}<0.001\right)$. This was found to be due to one sample area only, number 1 (see Fig. 1), where centroid size was significantly larger than in other localities (Tukey's a posteriori test, $\mathrm{P}<$ 0.001 in each three comparisons) (Fig. 3).

The first principal component explained $27.97 \%$, the second $23.95 \%$, the third $11.84 \%$, the fourth $9.44 \%$, and the fifth $8.54 \%$ of landmark shape variation. Thus, five most significant principal components explained $81.75 \%$ of the all shape variability. It seems evident that the genital outline shape shows substantial variation, but also 


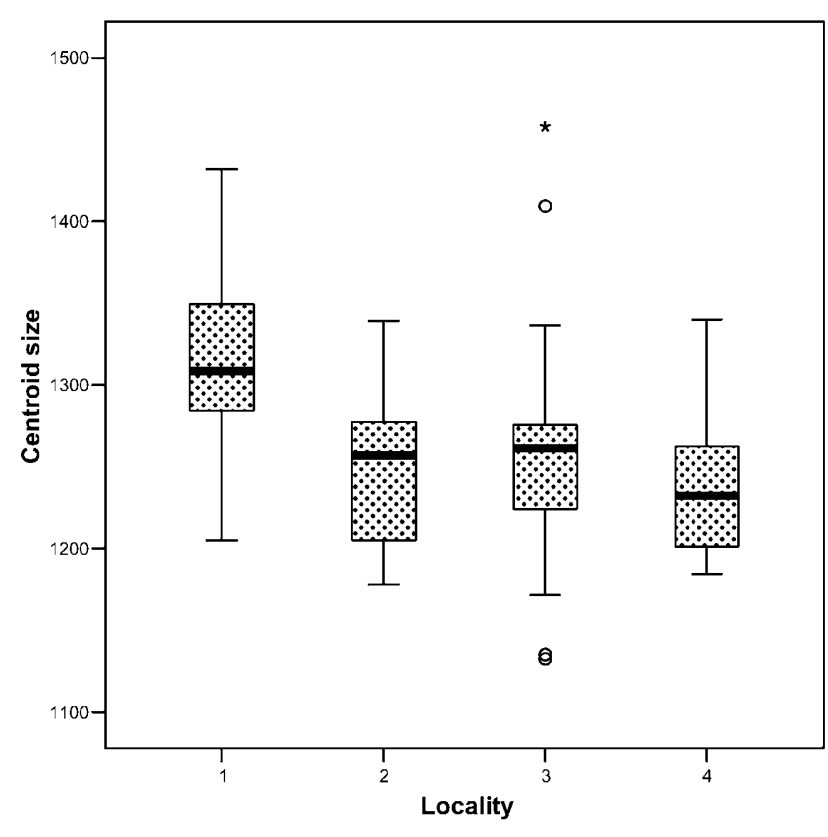

Fig. 3. Centroid sizes of genitalia for four geographical sample areas. The boxes indicate the interquartile ranges $(50 \%$ of all specimens) and the bars the full range, except for outliers. Outliers with values between 1.5 and 3 box lengths from the upper or lower edge of the box are indicated as circles and outliers with values more than 3 box lengths from the upper or lower edge of the box with asterisks.

that this variation seems continuous (Fig. 4). Informative shape variation is concentrated around the landmark 8 (the tip of the projection of valva), since the relative contributions of the landmarks 7-9 were clearly more prominent than those of other landmarks varying from $12.8 \%$ to 16.8\% (also see Fig. 5). Since Procrustes superimposition tends to spread localized variation across all landmarks ("Pinocchio effect"), and as this typically happens with elongated structures, the observed variation in the landmarks 7-9 probably represent the minimum limits of variation. There is, however, much variation in some other landmarks as well, particularly around the endpoints of the outline (Fig. 5). This variation is, however, of less information, with relative contributions of only $0.6-0.7 \%$, meaning that variation there is random and not correlated with variation of other landmarks. The relative contributions of the rest of the landmarks were $2-6 \%$ per landmark. The thin-plate spline-deformation visualization (shown with right valvae) reveals that the first component reflects the change in the steepness of the projection around the landmarks 7-9 (Fig. 6). The second component accounts the change in the orientation of the proximal part of valva in relation to its distal enlargement (Fig. 6). The third principal component accounts the relative size of the distal enlargement in relation the size of proximal half ("stalk") of valva; in one extreme the enlargement is about as large as the stalk, whereas in the other end the enlargement is only about half of the stalk area (Fig. 6).

We did not find evidence for geographic shape variation (Wilks' $\Lambda=0.291, \mathrm{~F}_{102,225.471}=1.127, \mathrm{P}=0.232$ ) sug-

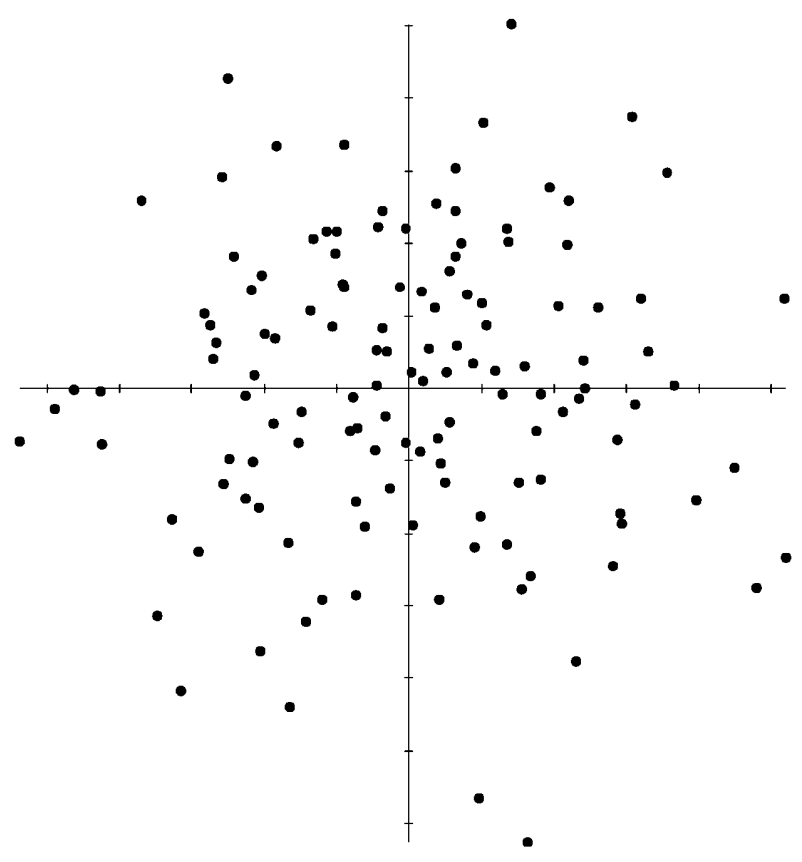

Fig. 4. Distribution of shape variation of right valvae of $P$. luedersiana specimens along the first (X axis) and the second (Y axis) principal components.

gesting that the extent and form of shape variation is relatively similar throughout the study area.

\section{Correlation between genital size and shape}

The regression analysis indicated that the shape of male genitalia shows correlation with genital size (Table 1). There is a significant dependence between size and the second component (Table 1), but no relationship between size and the first and third principal components.

\section{DISCUSSION}

We investigated the extent of genital variability in the male genitalia of the moth species $P$. luedersiana. Considering how frequently details of genital morphology are

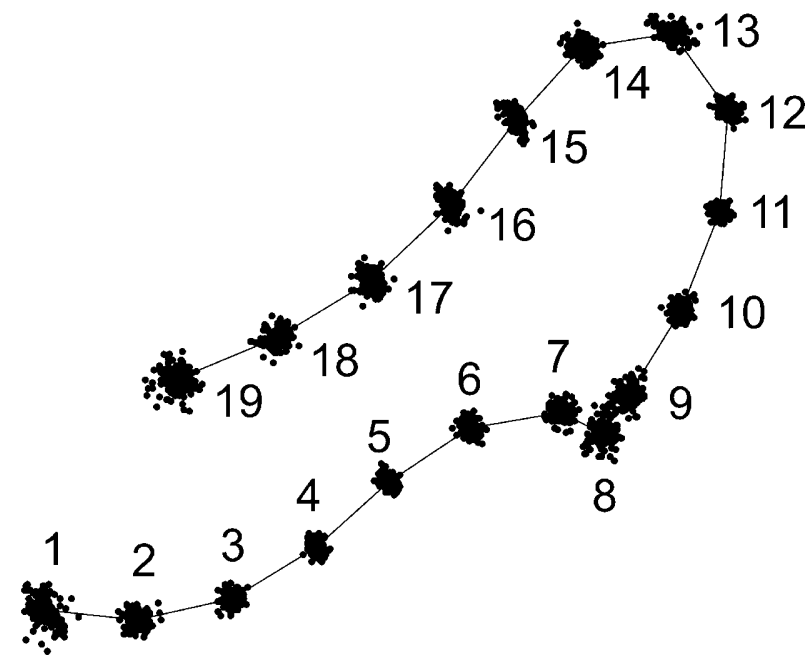

Fig. 5. Observed shape variation of each landmarks of the right valvae of $P$. luedersiana. Variation in scale, rotation and location are filtered from the data. 

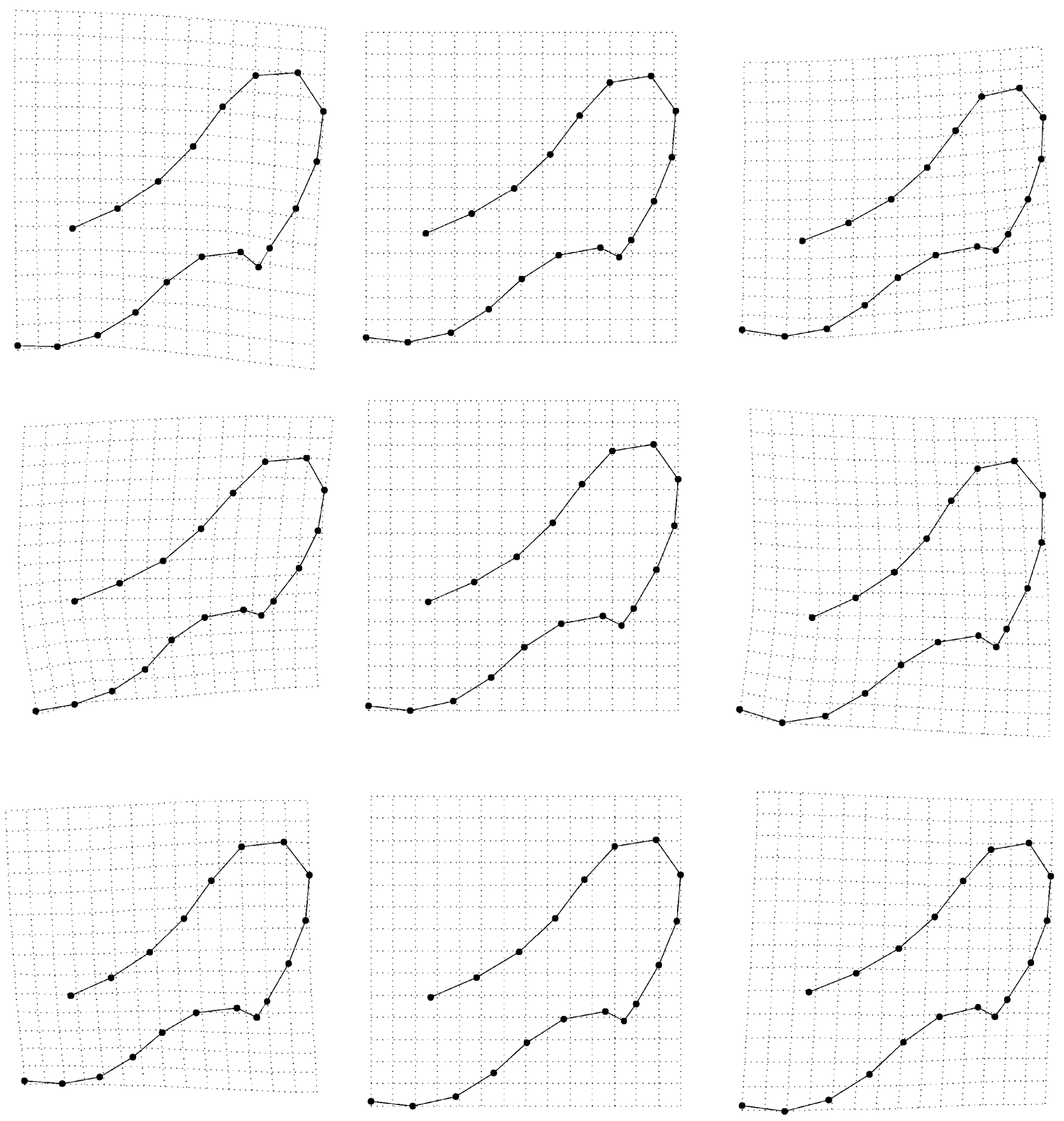

Fig. 6. Shape variation range of the most significant shape components of the right valvae of $P$. luedersiana, depicted with aid of thin-plate spline deformation grids. Top horizontal row: shape change along the PCA 1; mid row: shape change along the PCA 2; Bottom row: shape change along the PCA 3. The average (consensus) shape configuration is shown in the middle of each row.

used in taxonomy, such studies are still too rare. We found no evidence for discrete clusters. Extreme types represent borderlines of considerable, but continuous phenotypic variation. We also found that the genital size varies in a geographical scale: One population showed larger genitalia than others. Thirdly, we found that genital shape in not completely independent of size: One shape dimension showed slight but statistically significant correlation with genital overall size.

Although the study was initially motivated by a priori observation on genital shape variation in the target moth, there are no grounds to assume that it shows exceptional intraspecific genital variability. Rather, the presumption of lower degree of shape variation in genital traits in relation to non-genital traits is not supported by the many indepth case studies. For example, Arnqvist \& Thornhill (1998) and House \& Simmons (2003) found that the morphometric variability in genital traits of a water strider and a beetle, respectively, is of the same level as that is in non-genital traits. Similarly, the genital structures of Trichogramma wasps are shown to vary a lot and to be influenced by the host species (Pinto et al., 1989; Querino \& 
TABLE 1. Regression analysis results of correlation between genital centroid size and shape variables (PC1-PC3). The three principal components accounted the $64 \%$ of all observed shape variation.

\begin{tabular}{lcrc}
\hline Variable & $\begin{array}{c}\text { Right valva } \\
\text { coeff }\end{array}$ & $t$ & $P$ \\
\hline PC1 & -0.126 & -1.467 & 0.145 \\
PC2 & 0.216 & 2.513 & 0.013 \\
PC3 & -0.034 & -0.398 & 0.691 \\
\hline
\end{tabular}

n, F, P, R ${ }^{2} 131,2.876,0.039,0.064$

Zucchi, 2002). Some studies have shown that taxonomy based on the presumption of morphologic invariability has led to wrong taxonomic conclusions (Drotz et al., 2001; Mutanen, 2005). In both these cases, shape variation between "species" was shown to vary notably, but continuously in local or geographical scales. Sometimes, new species are described based on a single or few individuals, which are later found to represent extremes of a large intraspecific variability. Coleophora betulaenanae Klimesch, for instance, was described from a single male specimen (Klimesch, 1958), but an investigation of a large specimen number of the related $C$. vacciniella (H.-S.) revealed large continuous variation covering also the $C$. betulaenanae (genital) type (Itämies \& Tabell, 1997). The wide-spread presumption of low genital variability is patent in many handbooks, where genital differences of related species are presented and explained, but often based on scarce (or undefined) material. This easily leads to the situation highlighted by Itämies et al. (2003). They reported that none of the purported genital differences between closely related (but evidently separate) Aethes cnicana (Westwood) and A. rubigana (Treitschke) held true when large series were examined. Such problems are particularly common where differences are quantitative rather than qualitative in nature. Genital variation of $P$. luedersiana is in a good accordance with findings of most in-depth examinations of genital variability. It is evident that the shape of male genitalia of $P$. luedersiana expresses notable variability, which, using restricted material, could readily lead to its unjustified splitting into two or more genitally differentiated species. This observation is not in a good agreement with the lock-and-key hypothesis, but fits well with the predictions of sexual selection hypothesis (cf. Arnqvist, 1997). We, however, stress that we did not compare variation of genital traits with that of non-genital traits of $P$. luedersiana, and therefore this study cannot be treated as a powerful test in discriminating between genital evolution hypotheses. Such tests have generally indicated genital variation to be equal to variation of non-genital traits (e.g. Arnqvist \& Thornhill, 1998; Mutanen et al., 2006).

The frequent observation of large intraspecific genital variation seems to apply to shape only. Several studies in a wide variety of invertebrate taxa have convincingly shown the genital size to vary less than body size, therefore expressing negative allometry in relation to overall body size (Eberhard et al., 1998; Schmitz et al., 2000; Uhl \& Vollrath, 2000; Tatsuta et al., 2001; Bernstein \& Bern- stein, 2002; Kawano, 2004; Hosken et al., 2005; Mutanen et al., 2006). In some studies, reduced values of the coefficient of variation have been detected in genital traits as well (Eberhard et al., 1998; Teder, 1998; Hosken et al., 2005; Mutanen et al., 2006). However, as pointed out by Eberhard et al. (1998), the coefficient of variation may be affected not only by greater dispersion of individual points along the allometric line (usually the addressed question), but also by the slope itself. It is therefore evident that in many studies the low values of CV were the consequence of negative allometry. This problem illustrates the restrictions of the traditional metric approach in morphometric studies. Using the traditional approach, one cannot completely separate the effects of size and shape from each other. Furthermore, the traditional approach captures mostly variation in size, but limitedly that of shape. Since rapid and divergent genital evolution concerns mainly shape, geometric morphometrics should be preferred over this traditional approach.

$P$. luedersiana belongs to the large Lepidopteran family Tortricidae (leaf-rollers), with over 5000 described species worldwide (Scoble, 1995). Much attention has been paid to the pheromone compositions of the species in the tribe Grapholitini, because it includes many serious pest species (Witzgall et al., 1996). P. luedersiana belongs to that species-rich group. The group consists of many species which are difficult to identify externally, and for that reason both the male and the female genitalia, usually having good diagnostic features, are illustrated in handbooks (e.g. Razowski, 2001). In North Europe, pheromones have been used not only for pest control, but also for faunistic and taxonomic purposes. As a consequence, knowledge about the distribution and taxonomy of these species, which are difficult to observe using traditional methods, is increasing. Many predominantly rare species have shown to be common, and the taxonomic status of some have been either clarified or questioned. Our results indicate that genital morphology should not be carelessly treated as superior to other morphological characters in taxonomic studies. Genital traits show variation, and even though their size varies less than that of non-genitalian traits, spatial variation may occur also there. Thus, due to possible correlation between size and shape, species with remarkable variation in overall size may consequently exhibit larger genital variability.

ACKNOWLEDGEMENTS. We thank the staff of the Finnish Museum of Natural History, J.-P. Kaitila, and B. Wikström who kindly loaned material for us. We are deeply indebted to M. Hyvärinen, R. Tornberg, and P. Välimäki for valuable discussions and a number of useful comments on the paper. The study was financially supported by the Finnish Cultural Foundation.

\section{REFERENCES}

Adams D.C., Slice D.E. \& Rohlf F.J. 2004: Geometric morphometrics: Ten years of progress following the "revolution". Ital. J. Zool. 71: 5-16.

ARNQVIST G. 1997: The evolution of animal genitalia: distinguishing between hypotheses by single species studies. Biol. J. Linn. Soc. 60: 365-379. 
ARNQvist G. 1998: Comparative evidence for the evolution of genitalia by sexual selection. Nature 393: 784-786.

ARNQVIST G. \& MÅRTENSSON T. 1998: Measurement error in geometric morphometrics: empirical strategies to assess and reduce its impact on measures of shape. Acta Zool. 44: 73-96.

ArNQvist G. \& ThorNhill R. 1998: Evolution of animal genitalia: patterns of phenotypic and genotypic variation and condition dependence of genital and non-genital morphology in water strider (Heteroptera: Gerridae: Insecta). Genet. Res. 71: 193-212.

Bernstein S. \& Bernstein R. 2002: Allometry of male genitalia in a species of soldier beetle: Support for the one-size-fits-all hypothesis. Evolution 56: 1707-1710.

BooksteIN F.L. 1991: Morphometric Tools for Landmark Data. Geometry and Biology. Cambridge University Press, Cambridge, $435 \mathrm{pp}$.

BooKsTEIN F.L. 1997: Landmark methods for forms without landmarks: Localizing group differences in outline shape. Med. Image Anal. 1: 225-243.

Drotz M.K., SAura A. \& Nilsson A.N. 2001: The species delimitation problem applied to the Agabus bipustulatus complex (Coleoptera, Dytiscidae) in North Scandinavia. Biol. J. Linn. Soc. 73: 11-22.

Dufour L. 1844: Anatomie générale des Diptères. Ann. Sci. Nat. 1: $244-264$.

Eberhard W.G. 1985: Sexual Selection and Animal Genitalia. Harward University Press, Cambridge, xii +244 pp.

Eberhard W.G., Huber B.A., Rodriguez R.L.S., Briceno R.D., Salas I. \& Rodriguez V. 1998: One size fits all? Relationships between the size and degree of variation in genitalia and other body parts in twenty species of insects and spiders. Evolution 52: 415-431.

Hosken D.J. \& Stockley P. 2004: Sexual selection and genital evolution. Trends Ecol. Evol. 19: 87-93.

Hosken D.J., Minder A.M. \& Ward P.I. 2005: Male genital allometry in Scatophagidae (Diptera). Evol. Ecol. 19: 501-515.

House C.M. \& Simmons L.W. 2003: Genital morphology and fertilization success in the dung beetle Onthophagus taurus: An example of sexually selected male genitalia. Proc. R. Soc. Lond. (B) 270: 447-455.

ItÄMIES J. \& TABelL J. 1997: Variation in male genitalia of Coleophora vacciniella H.-S. (Lepidoptera, Coleophoridae). Entomol. Fenn. 8: 145-150.

Itämies J., Mutanen M. \& Lankinen P. 2003: Revision of the European Aethes rubigana complex with a description of genetically confirmed Aethes kyrkii sp. n. (Lepidoptera, Tortricidae). Insect Syst. Evol. 34: 3-14.

Kawano K. 2004: Developmental stability and adaptive variability of male genitalia in sexually dimorphic beetles. Am. Nat. 163: 1-15.

Kendall D.G. 1977: The diffusion of shape. Adv. Appl. Probab. 9: $428-430$.

Kendall D.G. 1984: Shape-manifolds, Procrustean metrics and complex projective spaces. Bull. Lond. Math. Soc. 16: 81-121.

KLIMESCH J. 1958: Coleophora betulaenanae n. sp. (Lepidoptera, Coleophoridae). Opusc. Zool. 12: 1-4.

Mutanen M. 2005: Delimitation difficulties in species splits: a morphometric case study on the Euxoa tritici complex (Lepidoptera, Noctuidae). Syst. Entomol. 30: 632-643.
Mutanen M. \& Kaitala A. 2006: Genital variation in a dimorphic moth Selenia tetralunaria (Lepidoptera, Geometridae). Biol. J. Linn. Soc. 87: 297-307.

Mutanen M., Kaitala A. \& MönKKönen M. 2006: Genital variation within and between three closely related Euxoa moth species: testing the lock-and-key hypothesis. J. Zool. 268: 109-119.

Pinto J.D., Velten R.K., Platner G.R. \& Oatman E.R. 1989: Phenotypic plasticity and taxonomic characters in Trichogramma (Hymenoptera: Trichogrammatidae). Ann. Entomol. Soc. Am. 82: 414-425.

QUERINO R.B. \& ZuCCHI R.A. 2002: Intraspecific variation in Trichogramma bruni Nagaraja, 1983 (Hymenoptera: Trichogrammatidae) associated with different hosts. Braz. J. Biol. 62: 665-679.

Razowski J. 2001: Die Tortriciden (Lepidoptera, Tortricidae) Mitteleuropas. F. Slamka, Bratislava, 319 pp.

Rohlf F.J. 2003: TpsRelw, Version 1.35. Department of Ecology and Evolution, State University of New York at Stony Brook. Available at http://life.bio.sunysb.edu/morph/

Rohlf F.J. 2004a: TpsDig, Version 1.40. Department of Ecology and Evolution, State University of New York at Stony Brook. Available at http://ife.bio.sunysb.edu/morph/

RohlF F.J. 2004b: TpsUtil, Version 1.26. Department of Ecology and Evolution, State University of New York at Stony Brook. Available at http://life.bio.sunysb.edu/morph/

RoHLF F.J. \& Marcus L.F. 1993: A revolution in morphometrics. Trends Ecol. Evol. 8: 129-132.

Rohlf F.J. \& Slice D.E. 1990: Extensions of the Procrustes method for the optimal superimposition of landmarks. Syst. Zool. 39: 40-59.

Schmitz G., Reinhold K. \& Wagner P. 2000: Allometric relationship between genital size and body size in two species of mordellid beetles (Coleoptera: Mordellidae). Ann. Entomol. Soc. Am. 93: 637-639.

Scoble M.J. 1995: The Lepidoptera: Form, Function and Diversity. Oxford University Press, Oxford, $404 \mathrm{pp}$.

ShAPIRO A.M. \& Porter A.H. 1989: The lock-and-key hypothesis: evolutionary and biosystematic interpretation of insect genitalia. Annu. Rev. Entomol. 34: 231-245.

Tatsuta H., Мizota K. \& Акімото S.I. 2001: Allometric patterns of heads and genitalia in the stag beetle Lucanus maculifemoratus (Coleoptera: Lucanidae). Ann. Entomol. Soc. Am. 94: 462-466.

TEDER T. 1998: Limited variability of genitalia in the genus Pimpla (Hymenoptera: Ichneumonidae): inter- or intraspecific causes? Neth. J. Zool. 48: 335-347.

Uhl G. \& Volrath F. 2000: Extreme body size variability in the golden silk spider (Nephila edulis) does not extent to the genitalia. J. Zool. 251: 7-14.

Witzgall P., Chambon J.-P., Bengtsson M., Unelius C.R., Appelgren M., Makranczy G., Muraleedharan N., Reed D.W., Hellrigl K., Buser H.-R., Hallberg E., Bergström G., Tóth M., Löfsted C. \& LöFQvist J. 1996: Sex pheromones and attractants in the Eucosmini and Grapholitini (Lepidoptera, Tortricidae). Chemoecology 7: 13-23.

Yezerinac S.M., Lougheed S.C. \& HandFord P. 1992: Measurement error and morphometric studies: statistical power and observer experience. Syst. Biol. 41: 471-482.

Zelditch M.L., Swiderski D.L., Sheets H.D. \& Fink W.L. 2004: Geometric Morphometrics for Biologists: A Primer. Elsevier Academic Press, New York, 443 pp.

Received April 7, 2006; revised and accepted October 18, 2006 